\title{
LA FORMACIÓN DE INTELECTUALES \\ INDIGENAS EN CONTEXTOS DE \\ INTERNACIONALIZACIÓN: ESTUDIO DE UN \\ CASO
}

Esteban Rodríguez Bastos ${ }^{1}$

\section{RESUMEN}

El articulo tiene como propósito presentar un avance de la investigación que actualmente realizo en el proceso de formación Doctoral, el cual se desarrolla en el contexto de internacionalización de las Instituciones de Educación Superior (IES) en México, la investigación se realizó en la Universidad Pedagógica Nacional de Colombia (UPN-C) por medio de la participación de estudiantes indígenas, académicos (tutores) $\mathrm{y}$ académicos (cotutores extranjeros), que participaron en el programa de movilidad internacional de la Maestría en Desarrollo Educativo (MDE) de la Universidad Pedagógica Nacional de México (UPN-M) impulsadas por el Consejo Nacional de Ciencia y Tecnología (CONACyT), es importante remarcar la formación de intelectuales indígenas a través de sus experiencias de movilidad internacional, por lo que presentaré algunas categorías que se están trabajando y experiencias que se generaron durante el desarrollo de su formación de investigadores complementada a partir de la vivencia de la movilidad y estancia académica internacional presentándolo bajo un estudio de caso.

PALABRAS-CLAVE: Internacionalización. Movilidad Internacional. Formación. Intelectuales Indígenas. Interculturalidad.

\section{ABSTRACT}

The article aims to present an advance of the research that I currently do in the Doctoral training process, which is developed in the context of internationalization of Higher Education Institutions (IES) in Mexico. (UPN-C) through the participation of indigenous students, academics

1 Grado de Maestría en Pedagogía otorgado por la UNAM México, Lic. en Sociología egresado de la Universidad Autónoma Metropolita campus Xochimilco (UAM-X). Actualmente es Doctorante de Pedagogía en la UNAM, académico-investigador en la Universidad Pedagógica Nacional y en la FES Aragón UNAM. Ciudad de México CDMX, México. E-mail: estirdi@gmail.com. 
(tutors) and academics (foreign contributors), who participated in the international mobility program of the Master's in Educational Development (MDE) of the University (UPN-M) promoted by the National Council of Science and Technology (CONACyT), it is important to highlight the training of indigenous intellectuals through their experiences of international mobility, so I will present some categories that are being worked on and experiences that were generated during the development of its training of researchers complements based on the experience of mobility and international academic stay presented under a case study.

KEYWORDS: Internationalization. International Mobility. Training. Indigenous Intellectuals. Interculturality.

\section{De donde nace todo}

No puedo dejar de compartir el proceso donde se gestó el presente proyecto, el dar cuenta de una experiencia de investigación que me ha llevado a dar un vuelco a mi vida y la cual no se inicia precisamente en mi lugar de trabajo, que es de donde aportaré el presente artículo, inicia en una experiencia propia como Misionero Laico $^{2}$ en la Selva Amazónica del Perú en donde destine algunos años de mi vida a trabajar con comunidades rivereñas e indígenas en actividades pastorales, educativas, defensoría de la vida, equidad de género, ecología y preservación del rio y la selva de la amazonia peruana a través de temas de sustentabilidad ambiental etc., dentro del Vicariato Apostólico de San José del Amazonas que abarca territorialmente de Iquitos Perú hasta las tres fronteras (Perú, Colombia y Brasil), y circunda con la frontera de ecuador.

La llegada a estas tierras, me invito el aceptar que mi identidad (la cual ya se encontraba convencida de saber muchas

2 La Constitución Dogmática Lumen Gentium afirma que la vocación del Misionero Laico consiste en "iluminar y organizar todos los asuntos temporales a los que están estrechamente vinculados, de tal manera que se realicen continuamente según el espíritu de Jesucristo y se desarrollen y sean para la gloria del Creador y del Redentor" (n. 31). El Decreto conciliar Apostolicam Actuositatem indica que forma parte de esta vocación el apostolado de los laicos a partir de aportar su profesión o conocimientos técnicos, relacionados con una vida de fe que puedan a portar mejoras a las comunidades donde se insertan. 
cosas de la vida) necesitaba de la apertura de los sentidos y humildad ante todo para poder aprender cosas nuevas, más que intentar enseñar. Esto implicaba iniciar una nueva etapa de aprender cómo encontrar el sentido a mis teorías apropiadas ante una nueva practica de vida en la selva, donde no había servicios propios de la urbe o la metrópoli como el servicio de luz, el agua potable, centros de salud, las comunicaciones son muy restringidas y al capricho del clima; donde el Estado no hace presencia para acompañar a sus comunidades a través de sus instituciones; en donde más que artefactos electrodomésticos se requiere de la habilidad humana y los saberes del diálogo con la naturaleza para poder vivir el día con día; en donde la explotación de los recursos naturales en la tala de árboles, la búsqueda y explotación de yacimientos petroleros y el narcotráfico son las opciones que hacen de los indígenas y rivereños sean empleados (esclavos) para poder sacar adelante a sus familias; en medio de esa realidad de la selva me pregunte ¿Cómo aprender a sobrevivir y como vivir dignamente en una cultura distinta a la nuestra? ¿Qué aprendizajes son los que más se valoran y como darles su justo valor? ¿Cómo legitimarlos para experiencias futuras? Etc.

\section{Reincorporación: encuentro con la Movilidad Internacional}

Al retornar a la Ciudad de México (CDMX) lugar donde vivo actualmente, tengo la necesidad de reinsertarme en la vida laboral y el destino me brinda la oportunidad de regresar a trabajar en la $\mathrm{UPN} \mathrm{M}^{3}$, lugar del cual renuncie antes de irme hacer la experiencia de Misionero Laico hace algunos años... hoy retornaba para incorporarme al espacio de los posgrados de la UPN-M, un espacio que cuenta con programas de doctorado, maestría y especialidades que son los programas que se ofertan y que constantemente buscan el

3 La Universidad Pedagógica Nacional de México es una institución pública de educación superior, creada por decreto presidencial el 29 de agosto de 1978. Tiene la finalidad de formar profesionales de la educación en licenciatura y posgrado para atender las necesidades del Sistema Educativo Nacional y de la sociedad mexicana en general. Ofrece, además, otros servicios de educación superior como diplomados y cursos de actualización docente, realiza investigación en materia educativa y difunde la cultura pedagógica, la ciencia y las diversas expresiones artísticas y culturales del país. 
reconocimiento de pertenencia a la lista de los Programas Nacionales de Posgrados de Calidad ${ }^{4}$ (PNPC) del Consejo Nacional de Ciencia y Tecnología ${ }^{5}$ (CONACyT) el cual dota de estímulos por parte del Estado para la institución y el desarrollo de cada uno de los actores, académicos, administrativos y estudiantes, otorgándoles a estos últimos la posibilidad de acceder a una beca de manutención y de movilidad nacional o internacional.

La UPN-M cuenta dentro de la oferta de programas con el de Maestría en Desarrollo Educativo el cual tiene como propósito: "Formar profesionales de alto nivel para desempeñarse en las variadas funciones educativas-docentes, pedagógicas, de gestión y administración, de planeación, de instrumentación y operación de políticas- requeridas en el Sistema Educativo Nacional, capaces de diseñar y llevar a cabo proyectos educativos de intervención, innovación y desarrollo, coadyuvando así a la comprensión y mejoramiento de prácticas educativas en el sector educativo" (revisado en el portal UPN-M (2018)).

Tener el reconocimiento como programa PNPC, se logra a través de un riguroso proceso de evaluación de pares académicos y se otorga a los programas que muestran haber cumplido con los más altos estándares de calidad y pertinencia. Para lograr lo anterior, se ha definido un conjunto de Políticas que orienta en desarrollo del PNPC:

- Impulsar nuevas formas de organización del posgrado para favorecer el desarrollo nacional en la sociedad del conocimiento.

- Incrementar la capacidad de absorción del conocimiento científico, tecnológico y de innovación en los sectores de la sociedad.

4 El PNPC: fomenta la mejora continua y el aseguramiento de la calidad del posgrado nacional, para incrementar las capacidades científicas, humanísticas, tecnológicas y de innovación del país, que incorporen la generación y aplicación del conocimiento como un recurso para el desarrollo de la sociedad y la atención a sus necesidades, contribuyendo así a consolidar el crecimiento dinámico y un desarrollo más equitativo y sustentable del país.

5 El Consejo Nacional de Ciencia y Tecnología (CONACyT) es un organismo público descentralizado del gobierno federal mexicano dedicado a promover y estimular el desarrollo de la ciencia y la tecnología en México. Tiene la responsabilidad oficial para elaborar las políticas de ciencia y tecnología nacionales". Revisado en la página de CONACyT (2018). 
- Posicionar el posgrado mexicano de alta calidad en el ámbito internacional. Revisado en el portal CONACyT (2018).

En la investigación me enfocare en el tercer punto de las políticas que orientan al desarrollo del PNPC, es donde se deslinda mi investigación y la misma se desarrolla a partir de posicionar el posgrado mexicano de alta calidad en el ámbito de internacionalización, ya que este proceso invita al desarrollo de movilidad de estudiantes, académicos y administrativos a la par de construir vínculos interinstitucionales para una formación que aporte elementos para la generación del conocimiento. Retomo mis preguntas clave de estancia en el Vicariato apostólico en Perú con las que regrese de mí experiencia, para poder indagar a los estudiantes que se enfrentan a una movilidad académica y que, para mi sorpresa, compartimos muchos puntos en común en su procesos de formación durante su estancia internacional, pero principalmente en su desarrollo por un semestre en una cultura académica y una cultura en general totalmente diferente a la suya. Esta información quedo asentada en la tesis que lleva como título: La movilidad académica internacional: como una experiencia dinamizadora de la formación en los posgrados.

Un primer momento fue indagar sus experiencias de formación ${ }^{6}$ durante la movilidad internacional vivida, algunos aportes del estudio de maestría mencionado me otorgan herramientas y datos los cuales hoy me tienen atrapado en el proceso de mi formación Doctoral, parte de la información me arrojo, donde se destaca que en el programa de Maestría en Desarrollo Educativo que durante tres convocatorias para la realización de Movilidad Internacional a tres generaciones de estudiantes (2008-2010) (2010-2012) y (20122014) los cuales se integran en sus 3 campos de formación y a sus 11 Líneas de Aplicación y Generación del Conocimiento (LGAC).

$6 \quad$ Las experiencias de formación durante la movilidad internacional vivida es un término que generé durante el desarrollo de mi tesis de maestría y el cual serán entendidas como: todas aquellas acciones que produjeron principios de subjetividad en los actores y que se plasmaron en nuevos aprendizajes externos a los estudiantes y que trascendieron como producto de las relaciones sociales que vivieron durante su estancia. Lo que diría Silvia Satulovsky (2009) "Como la operación de transformación de sí mismo". 


\section{Formación e intelectuales}

Los conceptos y teorías que serán parte de esta investigación, son producto del proyecto trabajado en la maestría y la suma de nuevas necesidades a la investigación doctoral generando las base de este nuevo proyecto, inicio con la formación, la cual se ve exigida por la necesidad de construir a generaciones del siglo XXI, que se caracterizan por vivir procesos de globalización, en donde las tecnologías cumplen un papel acelerador, por la capacidad de producción en bienes y servicios a la cual tienen que dar respuesta en la aldea global y esa globalización es con tintes como nos dice Edgar Morín:

globalización comprendida casi siempre para describir únicamente la mundialización de las dimensiones económica y tecnológica, aunque muchos críticos han señalado que la globalización es una dinámica multidimensional, es decir ecológica, cultural, política y social, todo ella en mutua interdependencia; aun así no deja de ser un término que pertenece a una visión unidimensional y reductiva del devenir del devenir humano del planeta (2003:79).

A las exigencias y condiciones que imperan en el mundo es importante que dejemos de pensar en una formación que solo se logre al interior de las instituciones, superar las barreras de la enseñanza con base en teorías sin la posibilidad de confrontarlas, experimentarlas y ponerlas a prueba para intuir y descubrir su eficiencia y valía por la sociedad, la cual da su aval ante la posibilidad de cubrir y dar respuesta a sus necesidades.

Así la formación implica la tarea de integrar los procesos filosóficos, culturales, económicos, políticos de acuerdo a las necesidades de las comunidades y a lo anterior aportare una primera aproximación de la formación que en palabras del Dr. Antonio Carrillo: "Es el resultado de experiencias de vida (positivas y negativas) que adquieren los sujetos a lo largo de su vida", la cual se complementara con el termino de formación que rescata el Dr. Gerardo Meneses de Honore: 
Honore supone la formación: como una marcha ascendente y progresiva hacia el desarrollo de los procesos reflexivos, de generación de imágenes y sensibilidades acotadas en la objetivación en el ser y el espacio y tiempo delinean. Para él la formación se refiere más específicamente a la búsqueda del cambio personal, que rompe las formas rígidas que bloquean el ser (2015:80).

El académico investigador Gilles Ferry los aporta él nos dice:

para realizar una formación no es más que, en el mejor de los casos una ocasión para formarse, aunque en realidad ninguna persona se forma a través de sus propios medios (Es necesario tener mediaciones), tampoco se puede formar por un dispositivo, ni por una institución ni por otra persona" por lo que "formarse no puede ser más que un trabajo sobre sí mismo, libremente imaginado, deseado y perseguido, realizado a través de los medios que se ofrecen o que uno mismo se procura (1990: 43).

Lo anterior me permitió construir el término de: Las experiencias de formación durante la movilidad internacional vivida, ya comentado.

Hablar de intelectuales me invita a retomar la obra de Antonio Gramsci, el que nos lleva por todo un proceso histórico, político y económico en el cual nos ayuda a tener en cuenta una opción de como comprender que los hombres, al actuar en el mundo, crean la cultura, que es la obra humana en la realidad natural. Para Gramsci todo ser humano es un intelectual, claro dividido en dos categorías muy precisas:

- Los intelectuales tradicionales.

- Los intelectuales orgánicos

En los intelectuales tradicionales enmarca a todos aquellos que se encargan de respetar los procesos de producción, los procesos de técnicos y que se forman siempre al interior de la Universidad, son modelos reproducidos y que van marcando el surgimiento de nuevos intelectuales según las necesidades sectorizadas o parciales para que respondan a esas necesidades productivas y de dominación.

Los intelectuales orgánicos son aquellos que más allá de los procesos técnicos y reproducidos buscan romper lo establecido a 
través del diálogo con la cultura donde se logre nos solo transformar la industria, si no la evolución de la sociedad civil contemplándola como agente que cambia y para eso es importante romper y dialogar constantemente con los nuevos procesos de estado a tal grado que para Gramsci el intelectual Moderno aterrice en que no sean simplemente escritores, sino directores y organizadores involucrados en la tarea práctica de construir la sociedad.

\section{Construyendo a partir de la teoría fundamentada}

La metodología que se emplea en la investigación es de corte cualitativo con base en la teoría fundamentada, que en palabras de Sandoval no dice que es: "una Metodología General para desarrollar teoría a partir de datos que son sistemáticamente capturados y analizados; es una forma de pensar acerca de los datos y poderlos conceptuar" (1997:71). Así las entrevistas, trayectorias y revisión de documentos con el fin de generar cruces de información con la ayuda de la teoría de análisis del discurso pretendo aportar los artefactos generados y que den cuenta de la formación de indígenas en espacios de movilidad internacional.

\section{Los estudiantes Indígenas y su participación en la movilidad}

Se destaca que la mayoría de los estudiantes que participan en las movilidades internacionales del programa de maestría en la UPN-M son estudiantes Indígenas de la línea de Diversidad Sociocultural y Lingüística; otra información grata de destacar es que de estos estudiantes de la línea que han participado son los primeros en titularse en tiempo y forma del programa de maestría, situación que ha impactado en las evaluaciones ante CONACyT logrando posicionarnos como un programa consolidado; y por último destacaría que estos estudiantes indígenas con sus proyectos se han destacado con reconocimientos a las mejores tesis, artículos publicados internacionalmente y una destacada participación dentro de un Colectivo de investigación de educación Bilingüe intercultural e interinstitucional donde participan la Universidad Nacional 
Autónoma de México (UNAM), la Escuela Normal Bilingüe Intercultural de Oaxaca (ENBIO), y la UPN-M.

Los estudiantes Indígenas han logrado destacar en la institución a partir de logros académicos y eso nos remite a pensarlos desde otra perspectiva, su constante participación en foros, coloquios, encuentros tanto nacionales como internacionales nos permiten reconocerlos como modelos de estudiantes para todas las generaciones que cursan estos estudios, ello me lleva a indagar acerca de su formación, acerca de la movilidad la cual ubico a la misma como un Artefacto académico (que más adelante explicare) destacando que convoca a indagar ¿Cómo impacto la movilidad académica internacional en su formación? ¿Por qué los estudiantes indígenas después de una movilidad primaria (al salir de sus comunidades) continúan experimentando ahora con una movilidad internacional al encuentro nuevas culturas académicas? ¿Cuál es su proceso de transformación ante las experiencias de interculturalidad vividas? Etc.

\section{La complejidad del estudio}

El estudio es complejo ya que el estudiante indígena vive una triple movilidad, la primera saliendo de sus comunidades para incorporarse a procesos formativos en las ciudades o metrópolis del país donde viven es decir ya viven una primera movilidad y estancia fuera de su cultura; la segunda es la que viven en su inscripción al programa de movilidad internacional y salir a una estancia de seis meses en una institución en el extranjero y a partir de su incorporación a la institución del país que los recibe; aquí en algunos casos inicia una tercera movilidad la cual consiste en movilizarse dentro del país que los acoge. En término de experiencias es una construcción constante y estas tres etapas de movilidad aunadas al referente del término "etnointelectual" entendido en el proceso formativo como la "Formación de Intelectuales" nos dice Pedro Canales Tapia:

ser etnointelectual reviste una contradicción flagrante y hasta ignominiosa. En gran medida esta tención nace de "figuras" o "representaciones" sociales que impulsan a la población a definir a este sujeto de acuerda con estereotipos. De este modo, no es posible la existencia de este grupo, pues los 
indígenas no construyen su saber y cosmovisión desde la modicidad científica con estas prefiguras, como herencia de la modernidad eurocentrista, sino a partir de la experiencia holística con la naturaleza y los antepasados, lo cual se aleja de la traducción cartesiana. Pablo Marimán hace alusión a esta tensión, cuando plantea que hay intelectuales indígenas que se valen del saber occidental para llevar a cabo sus labores, mientras otros desarrollan sus actividades a partir de los códigos del saber étnico comunitario, lo que el autor denomina Kimvm o conocimiento mapuche transmitido por los(as) antiguos(as) los(as) ancianos(as) los(as) sabios(as) (Formación de intelectuales, 86).

Si alternamos o hacemos un cruce del desarrollo formativo entre los tres momentos de la movilidad académica internacional (salida de comunidad, estancia de movilidad internacional, e inserción a las culturas internas del país de estancia) y el procesos de formación de intelectuales indígenas, en los cuales la investigación se adentra en el proceso y choque de la formación occidentalizada en la que las universidades en su mayoría se ven inmersas, produce la necesidad de generar modelos de educación que propicien espacios de dialogo ya que los indígenas no aceptan del todo los procesos formativos occidentalizados y por ende se ven invitados a experimentar desarrollar sus propios procesos formativos que los ayude a sobrevivir en medio de culturas distintas a las de su origen.

En este momento me gustaría aporta un cuadro donde podremos apreciar como una estancia de movilidad internacional acciona, fue construida en el marco de la teoría de Alan Touraine de la construcción de sujetos (Ver cuadro 1). 
Cuadro 1 - Referentes teóricos con base a la teoría de sujetos de Alan Touraine.

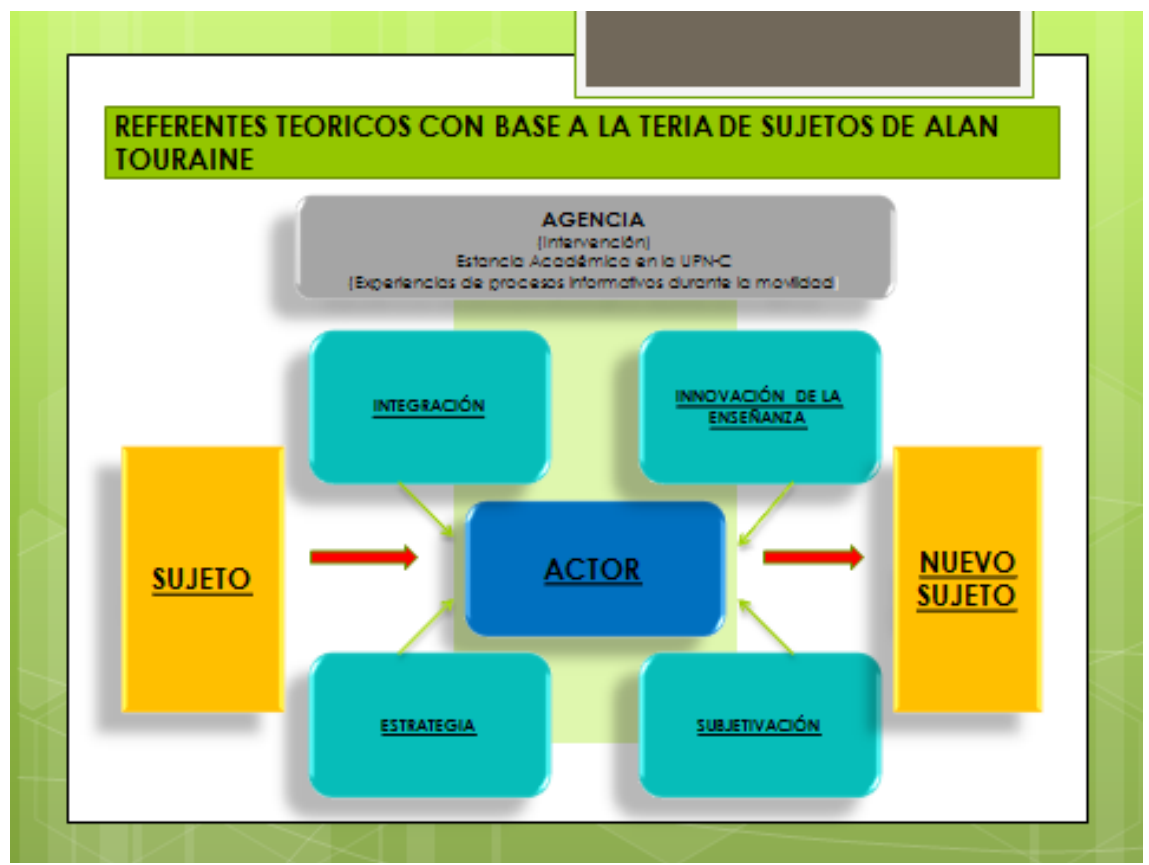

Fuente: Esteban Rodríguez Bustos.

El procesos enmarca la movilidad internacional que todo estudiante vive y el mismo nos señala que el sujeto es el estudiante que toma la decisión de realizar una estancia de movilidad internacional, en el momento en que es aceptado se transforma en actor de un nuevo proceso formativo en una cultura académica diferente, este actor vive procesos de integración a la nueva cultura académica vista a través de una interculturalidad en donde es un encuentro y vivencia entre culturas en la que es recibido y de ello nos dice:

Primera experiencia.

Toda experiencia es rica en cuanto conoces y convives con persona de diferentes contextos a los tuyos, experimentas otras formas de pensamiento y en la cuestión académica otras formas de conocimiento". (Estudiante de la generación 2012-2014 de la MDE).

En lo social la música, los espacios, los lugares con los que te encuentras con personas te hacen sentir en un ambiente más de confianza. En Colombia a mí me 
tocó ver que la gente ahí, no sé si por las condiciones de violencia que se viven y que vivieron en el pasado, pero son personas muy humildes, son personas muy accesibles, muy sociales y sociables, que te hacen sentir en confianza en su país, claro que eso no quiere decir que ya este pintado Colombia de color de rosa, si no también tienen sus índices de violencia, hay razones para los que lo hacen, pero en el ambiente social ahí las personas son muy amables y cuentan con lugares muy bonitos, que tú puedes experimentar al salir y conocer, que te sirven como el espacio de relajamiento, para que tampoco te sientas como asediado de tanto trabajo académico" (Estudiante de la generación 2012-2014 de la MDE).

Todo podría parecer igual en los países de América Latina, pero los signos y símbolos desde el lenguaje hasta la cultura escrita son un mundo que nos divide, pero que a la par nos invitan a descubrir y renovar procesos de sociedades en las que nos insertamos, a la par el termino enseñanza-aprendizaje nos invitan a insertarnos a partir de referentes críticos que contemplan la historia, que contemplan la música y sus referentes culturales que dan una identidad a las personas y que se enmarcan del procesos con el cual los estudiantes se insertan en las comunidad que en medio de experimentar la humildad y la confianza que generan los colombianos eso le ayudan a destacar valores que ayudan a su integración.

Parte de la experiencia del estudiante nos aporta de que la innovación de la enseñanza es un elemento que constantemente aparece como parte de un dialogo de culturas académicas así las experiencias de aprendizaje dan cuenta de ello:

Segunda experiencia.

En Colombia a mí me gustó mucho la movilidad, por las teorías que manejan, por el sentido crítico con el que desarrolla la educación, hablo de la universidad. Desde mi punto de vista esas experiencias, constituyen una base para generar otras propuestas metodológicas de investigación, otras propuestas didácticas de trabajo de campo, de trabajo de aula, porque la dinámica en la que se trabaja o en la que se trabajan los maestros, los docentes, con los que estudie allá en Colombia, es distinta a la que se trabaja en la UPN 
México. Aquí en México es el texto, la discusión a modo de seminario y la sesión se termina; allá en Colombia es el texto, el seminario, la discusión del seminario, pero además esa parte didáctica en la que se hace práctica el conocimiento" (Estudiante de la generación 2012-2014 de la MDE).

En el texto anterior se aprecia como el estudiante vive un proceso comparativo de la educación y también nos indica como esta didáctica le permite una apropiación y el despertar de un sentido crítico de ambas culturas, lo que para él le permite ver referentes innovadores en una práctica comparada de enseñanza aprendizaje, en la metodología, en la creación de propuestas didácticas para la enseñanza, en el trabajo de campo, en el trabajo del aula, todos estos procesos en un acercamiento comparativo entre las dos universidades UPN-M y UPN-C.

Las estrategias que desarrolla el estudiante es una constante y para muestra el siguiente texto:

Tercera experiencia:

Otra es la apertura al dialogo, en Colombia los asesores más allá de sentirse sujetos que saben más que tú y por ser académicos que por tener niveles de estudio más avanzados como posdoctores, son personas humildes que te dejan pensar a tu propio criterio, te dejan libre a tu criterio y lo único que te dicen es: bueno pues si piensas así, pues trata de sustentar lo que estás diciendo y aquí están estas lecturas, revísalas y ya después las platicamos, pero más allá de decir: "No, no es así", no nunca nos dijeron eso, al contrario que bueno que piensas así, que bueno que tienes esta idea y yo creo que con estos textos vas a fortalecer lo que tú conoces, entonces esa humildad de los asesores, esa colaboración de los asesores, como que te permite darte cuenta de donde estas tu equivocado, así te dan la autonomía como estudiante, como universitario, entonces esa autonomía también en su momento llega a formar parte de esa identidad a través de la lectura que se vuelve en tu formadora.

En esta parte de la entrevista se aprecia al dialogo como un eje constructor de pensamiento, de autonomía, de disciplina y a final de cuentas construye el sentido de la lectura como en 
elemento identitario del formado, sin necesidad de imposiciones, antes bien como una necesidad para consolidar las ideas expuestas del estudiante, para argumentar, para consolidar el su pensamiento crítico.

Y por último el sentido de subjetivación en el cual se relaciona con los procesos de interculturalidad que se viven y estos se reproducen constantemente derivado de la interrogante ¿Qué entiendes por la interculturalidad a partir de tu experiencia de Movilidad? Nos responde:

Cuarta experiencia:

Creo que ayer en mi examen lo decía y lo vuelvo a recalcar, el concepto como tal, no me voy a remitir al concepto, pero si tengo que hablar del concepto como tal como se entiende desde lo que lees, la interculturalidad como tal dices es un encuentro, es una vivencia entre culturas donde inicias una forma de relacionarse con los demás.

Catherine Walsh dice que como la interculturalidad está muy definida como concepto, está muy sesgada como concepto, pero hace falta que esa interculturalidad en las culturas no existe, sin embargo existe en los procesos, pero no como lo definen los intelectuales, Fidel Tubino por ejemplo, que es un especialista y hace una reflexión no solo teóricas, si no filosóficas de la interculturalidad y a partir de esos referentes que de los que plantea Catherine, lo que plantea Fidel Tubino, lo que plantea Boaventura de Sousa Santos, a partir de la lectura de estas personas de un gran conocimiento que no definen el conocimiento de interculturalidad, si no debaten la interculturalidad. Me ceo yo la idea de que la interculturalidad es una condición humana, atraviesa una línea horizontal que no solo se reduce a la convivencia, al encuentro, a la relación armónica como dicen otros, sino la interculturalidad atraviesa una línea de conocimiento, de saber, de poder, de ser, que tiene que ver no solo con un encuentro, una convivencia, una armonía, sino que tiene que ver con una relación del ser humano social, cultural y natural y esa condición humana apertura de la sensibilidad de los demás. 
La experiencia nos permite apreciar como el estudiante en movilidad, ya inicia un trabajo de manejo de conceptos y razonamiento crítico sobre el proceder de los autores y sus alcances en torno al concepto.

\section{Conclusiones}

A partir de esta primera aproximación observo que todo renace en la persona a partir de nuestras experiencias, de la capacidad de emplearlas para dar un sentido, se renuevan la identidad y sabiduría a nuestras decisiones, y eso sucedió en la selva amazónica del Perú y el estudiante en estancia en la UPN-C, donde la estancia ayuda a romper los referentes e iniciar una nueva etapa de procesos de aprendizaje para insertarme a una cultura diferente y en donde las sociedades nos enseñan a caminar con ella a través de sus saberes y cosmovisiones, para lograr sobrevivir en un mundo de explotación a las comunidades rivereñas e indígenas a través de procesos de explotación de este mundo globalizado, en el caso de la UPN-C para lograr procesos formativos en medio de culturas académicas diferentes y lo vivido nos permite ver con otros ojos la importancia de la movilidad internacional, permitiéndonos construir nuevos planteamientos que nos aproximen a dialogar con la subjetividad de las experiencias.

Con base en la movilidad, al encuentro, al intercambio y a la construcción de experiencias podemos ver como los estudiantes indígenas se han empoderado de los procesos de formación en ambientes de internacionalización y eso les ha permitido construirse y adquirir procesos de autonomía que se ven reflejados en sus logros, en sus cumplimientos, pero más allá de cumplir por cumplir, se aprecia en una primera aproximación los empoderamientos de discursos propios realizados bajo el encuentro de teorías y como resultado escriben tesis, escriben artículos, escriben libros, participan en congresos en otros países y ello nos invita a pensar que la movilidad a nivel de la educación superior (Aclarando que desde la educación media superior muchos países inician sus procesos de movilidad internacional, pero casi siempre enfocados en el aprendizaje de idiomas) implica la vivencia de experiencias y un procesos de interculturalidad en los sujeto y las culturas. 
Los sujetos que viven una movilidad internacional académica, entran en un proceso de formación de intelectuales indígenas, si hacemos un recuento de ambos términos apreciamos a lo que nos enfrenta este estudio en el sentido de que las experiencias de movilidad vividas van otorgando a la movilidad internacional el carácter de artefacto académico, en sentido de que todo artefacto es un detonador de cambio y transformación, de rompimiento y reconstrucción, propiciar como se forman nuevos sujetos, pero a la par se construyen nuevas metodologías, un pensamiento crítico y autocritico, que nos conducen a una autonomía en la toma de decisiones para la investigación que ayude a reconstruir o construir a las sociedades.

El estudio tendrá que desarrollar todo un procesos epistémico a la par de los estudiantes, para el cruce de las participaciones a través de las intervención del tutor y el cotutor, lo que nos permitirá ver la influencia de estos sujetos en el procesos formativo, y poder describir su labor formativa y probablemente logremos apreciar un modelo para la formación de intelectuales indígenas a través de la vivencia de una movilidad internacional, lo repito empleando el modelo de estancias de movilidad internacional como artefactos académicos.

\section{Referencias}

Concilio Vaticano II (1964). Constitución Dogmática sobre la Iglesia (Lumen Gentium). Roma.

Concilio Vaticano II (1965). Decreto sobre el apostolado de los Seglares (Apostoicam Actuositatem). Roma.

Ferry, G. (1993). Pedagogía de la formación. Eds. FFL-UBA. Novedades Educativas.

Gramsci, A. (2007). La alternativa pedagógica. México.

Marimán, P. (2010). Formación de intelectuales indígenas: ¿El rol de la educación superior? Ponencia presentada en el congreso de "Equidad, interculturalidad y educación superior" Temuco Chile.

Meneses, G. (2015). Formación y Pedagogía. España lucerna DIOGENESIS.

Morín, E. (2003). Educar en la era planetaria. Gedisa, España. 
Rodríguez, E. (2016). La movilidad académica internacional: como una experiencia dinamizadora de la formación en los posgrados. Tesis, UNAM, México. Revisado en la página de la UPN (2018). Disponível em: $<$ http:// www.upn.mx/index.php/estudiar-en-la-upn/posgrados/18-estudiar-en-la-upn/113-maestria-en-desarrollo-educativo) $>$. Revisado en pagina de CONACyT (2018) www.conacyt .gob.mx.

Sandoval, C. (1997). Investigación Cualitativa. Programa de Especialización en teoría, métodos y técnicas de investigación social. Medellín.

Zapata, C. (2007). Intelectuales indígenas piensan en América Latina. Editorial Abyala. Quito ecuador.

Recebido para publicação em maio de 2018. Aceito para publicação em junho de 2018. 